\title{
Chemoprotective Antimalarial Activity of P218 against Plasmodium falciparum: A Randomized, Placebo-Controlled Volunteer Infection Study
}

\author{
Mohamed Farouk Chughlay, ${ }^{1 \star}$ Myriam El Gaaloul, ${ }^{1}$ Cristina Donini, ${ }^{1}$ Brice Campo, ${ }^{1}$ Pieter-Jan Berghmans, ${ }^{2}$ Alexander Lucardie,${ }^{2}$ \\ Michael W. Marx, ${ }^{3}$ Mohammed H. Cherkaoui-Rbati, ${ }^{1}$ Grant Langdon, ${ }^{4}$ Iñigo Angulo-Barturen, ${ }^{5}$ Sara Viera, ${ }^{5}$ Anna Rosanas-Urgell, ${ }^{6}$ \\ Jean-Pierre Van Geertruyden, ${ }^{7}$ and Stephan Chalon ${ }^{1}$ \\ ${ }^{1}$ Medicines for Malaria Venture, Geneva, Switzerland; ${ }^{2}$ SGS Life Sciences, Antwerp, Belgium; ${ }^{3}$ ICON Clinical Research GmbH, Langen, Germany; \\ ${ }^{4}$ PTx Solutions, London, United Kingdom; ${ }^{5}$ GlaxoSmithKline, Tres Cantos, Madrid, Spain; ${ }^{6}$ Institute of Tropical Medicine, Antwerp, Belgium; \\ ${ }^{7}$ University of Antwerp, Antwerp, Belgium
}

\begin{abstract}
P218 is a highly selective dihydrofolate reductase inhibitor with potent in vitro activity against pyrimethamineresistant Plasmodium falciparum. This single-center, randomized, double-blind, placebo-controlled phase lb study evaluated P218 safety, pharmacokinetics, and chemoprotective efficacy in a $P$. falciparum sporozoite (PfSPZ) volunteer infection study (VIS). Consecutive dose safety and tolerability were evaluated (cohort 1), with participants receiving two oral doses of P218 1,000 mg 48 hours apart $(n=6)$, or placebo $(n=2)$. P218 chemoprotective efficacy was assessed (cohorts 2 and 3$)$ with direct venous inoculation of 3,200 aseptic, cryopreserved PfSPZ (NF54 strain) followed 2 hours later with two P218 doses of $1,000 \mathrm{mg}$ (cohort 2, $n=9$ ) or $100 \mathrm{mg}$ (cohort 3, $n=9$ ) administered 48 hours apart, or placebo $(n=6)$. Parasitemia was assessed from day 7 using quantitative PCR targeting the var gene acidic terminal sequence (varATS qPCR). By day 28 , all participants in cohort 2 (P218 1,000 mg) and 8/9 in cohort 3 (P218 $100 \mathrm{mg}$ ) were sterilely protected post-PfSPZ VIS, confirming P218 P. falciparum chemoprotective activity. With placebo, all six participants became parasitemic (geometric mean time to positive parasitemia 10.6 days [90\% Cl: 9.9-11.4]). P218 pharmacokinetics were similar in participants with or without induced infection. Adverse events of any cause occurred in 45.8\% (11/24) of participants who received P218 and $50.0 \%$ (4/8) following placebo; all were mild/moderate in severity, transient, and self-limiting. There were no clinically relevant changes in laboratory parameters, vital signs, or electrocardiograms. P218 displayed excellent chemoprotective efficacy against $P$. falciparum with favorable safety and tolerability.
\end{abstract}

\section{INTRODUCTION}

Children younger than 5 years bear the greatest mortality burden from malaria, accounting for $67 \%$ of the 405,000 recorded deaths in $2018 .^{1}$ Pregnant women are also vulnerable to malaria, with increased risk of low birth weight, perinatal/neonatal mortality, and continuing problems with child growth and cognitive development. ${ }^{1-3}$ Furthermore, malaria infection in sub-Saharan Africa among school-age children represents an important source of Plasmodium falciparum human-to-mosquito transmission. Preventive treatment in this group significantly decreases $P$. falciparum prevalence, anemia, and the risk of subsequent clinical malaria. $^{4}$

Sulfadoxine-pyrimethamine is recommended as malaria chemoprotection for the intermittent preventive treatment of pregnant women and infants,, ${ }^{5,6}$ and in combination with amodiaquine for seasonal malaria chemoprotection in children. ${ }^{7}$ However, sulfadoxine-pyrimethamine efficacy is threatened by drug resistance conferred by mutations in genes for the enzymes targeted by pyrimethamine ( $P$. falciparum dihydrofolate reductase [PfDHFR]), and sulfadoxine (dihydropteroate synthase). ${ }^{8,9}$ Given the need to reserve artemisininbased combination therapies for uncomplicated malaria, novel chemoprotective drug therapies are needed to defend vulnerable populations.

P218 is a highly selective PfDHFR inhibitor, with correspondingly robust in vitro and in vivo activity against $P$. falciparum, including pyrimethamine-resistant strains. ${ }^{9}$ In blood-stage malaria models, the P218 effective dose that

* Address correspondence to Mohamed Farouk Chughlay, Medicines for Malaria Venture (MMV), P.O. Box 1826, 20 Route de Pré-Bois, Geneva 1215, Switzerland. E-mail: chughlayf@mmv.org killed $90 \%$ of parasites (ED 90 was $0.75 \mathrm{mg} / \mathrm{kg}$ against Plasmodium chabaudi in CD-1 mice, and $1 \mathrm{mg} / \mathrm{kg}$ against a $P$. falciparum quadruple mutant in severe combined immunodeficient (SCID) mice humanized with circulating human red blood cells. ${ }^{9}$ However, P218 is of particular interest as a potential chemoprotective agent; in a liver-stage screening assay, the half-maximal effective concentration $\left(E_{50}\right)$ was $<0.012 \mu \mathrm{M}$ against $P$. falciparum schizonts. ${ }^{10}$

Safety is a key consideration for chemoprotection, and single oral doses of $10-1,000 \mathrm{mg}$ of P218 were well tolerated in a first-in-human clinical trial. ${ }^{11}$ Adverse events occurred in 15/ 64 participants, all of which were of mild severity, with no clinically relevant abnormalities in electrocardiogram (ECG), vital signs, or laboratory tests. ${ }^{11}$ Plasmodium falciparum dihydrofolate reductase inhibitors can deplete folate levels, ${ }^{12}$ but there was no evidence of a clinically important effect on folate with P218. ${ }^{11} \mathrm{P} 218$ was rapidly absorbed with a median $T_{\text {max }}$ of 1 hour post-dose and a mean half-life of 19.6 hours following a single $1,000 \mathrm{mg}$ dose. ${ }^{11}$ Three P218 primary metabolites were identified: $\mathrm{P} 218 \beta$-acyl glucuronide, $\mathrm{P} 218-\mathrm{OH}$, and $\mathrm{P} 218-\mathrm{OH} \beta$-acyl glucuronide, which also have antimalarial activity. ${ }^{11}$ Drug exposure increased dose proportionally between $100 \mathrm{mg}$ and $1,000 \mathrm{mg}$ for P218 and metabolites, with no effect of food on pharmacokinetics (PK). ${ }^{11}$ Thus, information to date suggests that $\mathrm{P} 218$ has activity and safety criteria consistent with a chemoprotective agent. ${ }^{9-11}$ The next step is to validate these findings in human participants exposed to P. falciparum.

The $P$. falciparum sporozoite (PfSPZ) volunteer infection study (VIS) is a valuable early drug development tool for exploring antimalarial and vaccine efficacy. ${ }^{13-16}$ Aseptic, cryopreserved PfSPZ are administered by direct venous injection to nonimmune human volunteers, with chemoprotective efficacy assessed as the capacity to prevent emergent parasitemia. ${ }^{15,17}$ 
The current study was conducted in nonimmune healthy participants to evaluate P218 safety and tolerability and chemoprotective efficacy using the PfSPZ VIS.

\section{MATERIALS AND METHODS}

Study design and ethics. This single-center, randomized, double-blind, placebo-controlled phase lb study was conducted between November 16, 2018 and June 3, 2019 at the SGS Phase 1 Clinical Unit, Ziekenhuisnetwerk Antwerpen (ZNA), Antwerp, Belgium. The study adhered to the Declaration of Helsinki, Guidance on Good Clinical Practice, and applicable local requirements. Ethical approval was obtained from the Commissie voor Medische Ethiek ZNA Institutional Review Board, Antwerp, Belgium. All participants provided written informed consent before study participation. The study was overseen by a safety review committee, blinded to treatment allocation, with protocol-defined meetings held at each study stage before progression.

The study comprised three cohorts, enrolled sequentially and randomized 3:1 to either P218 or placebo (Figure 1). The randomization list was prepared before study start by SGS Life Sciences Secure Data Office using SAS ${ }^{\circledR}$ software (SAS Institute Inc., Cary, NC). Randomization was balanced using randomly permuted blocks across the treatment groups.

Dose selection rationale. Dose selection used a population PK (popPK) model, together with in vivo efficacy data against $P$. falciparum blood stages from a humanized mouse model. The P218 minimum inhibitory concentration (MIC) was determined in the standard 4-day efficacy test in female NODscid IL-2RY ${ }^{\text {null }}$ mice with circulating human red blood cells infected with $P$. falciparum Pf3D7 ${ }^{0087 / N 9} .^{18,19}$ As a conservative approach, it was assumed that P218 in vivo activity in $P$. falciparum liver stages was at minimum equivalent to that observed against blood stages. The popPK model was used to simulate doses and regimens that would maintain P218 concentrations in human plasma above the target MIC for 6 days. This profile would provide effective chemoprotection across the entire $P$. falciparum liver-stage development cycle. The final model predicted that target P218 concentrations for chemoprotection would be achieved with two 1,000 mg doses administered 48 hours apart (cohort 2). A P218 dose of two $100 \mathrm{mg}$ doses administered 48 hours apart was predicted by the model as the lowest efficacious dose (cohort 3). The only available human safety data were from the first-in-human study, which evaluated a single 1,000 mg P218 dose. ${ }^{11}$ Thus, safety evaluation of the $1,000 \mathrm{mg}$ repeat dose with the

Cohort 1

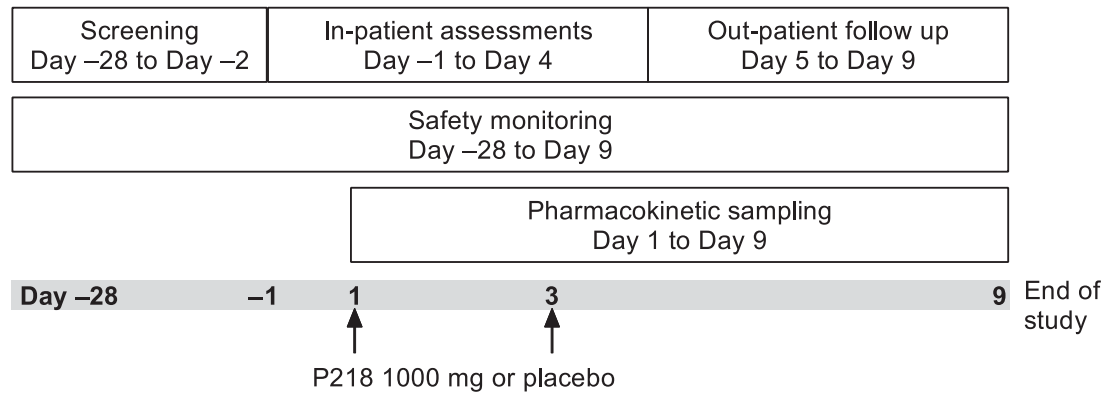

Cohorts 2 and 3

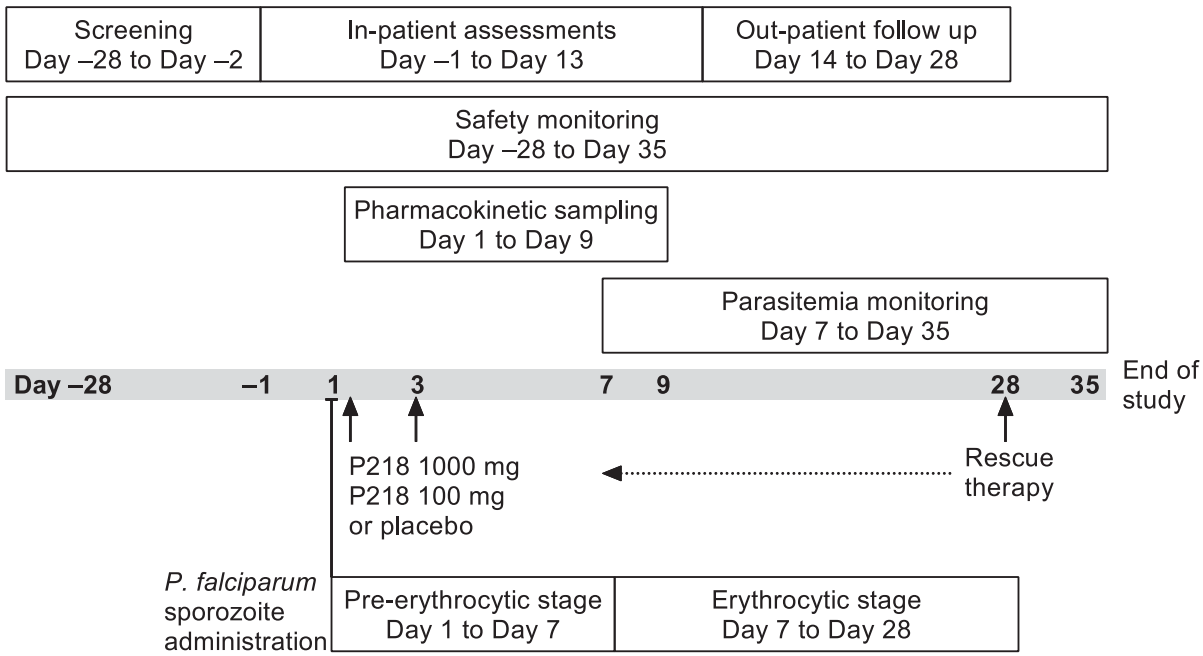

FIGURE 1. Study design for cohort 1, and cohorts 2 and 3. 
intended regimen was required in participants without $P$. falciparum infection (cohort 1), before evaluation in participants infected with $P$. falciparum. Further details of the popPK model are provided in the Supplemental Material (Methods S1).

Treatment. The primary objective of cohort 1 was to evaluate safety and tolerability for two consecutive 1,000 mg P218 doses administered 48 hours apart $(n=6)$ versus placebo $(n=$ 2) without PfSPZ inoculation. A sentinel dosing strategy was applied with two participants enrolled initially (P218 $n=1$; placebo $n=1$ ). After at least 24 hours following the final P218 dose and contingent on a review of safety data, a further six participants were enrolled (P218 $n=5$; placebo $n=1)$.

Cohorts 2 and 3 were designed to evaluate P218 chemoprotective activity in the PfSPZ VIS in 24 participants randomized 3:1 to either P218 or placebo. Cohort 2 included 12 participants who received either two $1,000 \mathrm{mg}$ P218 doses 48 hours apart $(n=9)$ or placebo $(n=3)$. The cohort was initiated with two participants (P218 $n=1$; placebo $n=1$ ) and progressed at least 24 hours after the last dose and following a safety review, to include five further participants (P218 $n=4$; placebo $n=1$ ), and then another five using the same procedure (P218 $n=4$; placebo $n=1)$. Cohort 3 included 12 participants who received either two $100 \mathrm{mg}$ doses of P218 48 hours apart $(n=9)$ or placebo $(n=3)$. Cohort 3 was initiated with six participants, with the remaining six enrolled at least 24 hours after the last dose and following a safety review.

Participants. Eligible participants were male or female, aged between 18 and 45 years with a body weight $\geq 50 \mathrm{~kg}$ and body mass index $19-30 \mathrm{~kg} / \mathrm{m}^{2}$. Participants had to be in good general health without clinically relevant medical illness, abnormal physical examination, ECG, or laboratory findings. Females had to have a negative pregnancy test and not be breastfeeding. Females of childbearing potential had to agree to use highly effective contraception from the screening visit to until $\mathbf{4 0}$ days after the last study dose. Males with a female partner of childbearing potential had to agree to use birth control from the day of the first study dose until 100 days thereafter. For full inclusion/exclusion criteria, see the Supplemental Material (Methods S2).

Procedures. Study medication was administered orally with $240 \mathrm{~mL}$ water after at least 8 hours of fasting. In cohorts 1 and 2, four matched $250 \mathrm{mg}$ capsules of placebo or P218 were administered, and in cohort 3 , two matched $50 \mathrm{mg}$ capsules of placebo or P218 were taken. After administration, a minimum 2-hour fast was required, followed by standard meals.

For cohort 1, participants were admitted to the clinical unit in the morning of day -1 , with first administration of P218 or placebo on day 1 , and second administration 48 hours later on day 3 (Figure 1). Participants were confined to the unit until day 4 (i.e., 36 hours after the second P218 administration) for close safety monitoring. After discharge from the clinical unit on day 4 , participants were followed up with daily outpatient visits until day 9 .

For cohorts 2 and 3, participants were admitted to the clinical unit in the morning of day -1 . Plasmodium falciparum sporozoite VIS was performed on day 1, consisting of 3,200 aseptic, cryopreserved PfSPZ (NF54 strain; Sanaria, Rockville, $M D$ ) inoculated by direct venous injection, as previously described. ${ }^{15,17}$ P218 or placebo was administered 2 hours after PfSPZ inoculation on day 1. The 2-hour delay was necessary to allow parasite migration to the liver, to avoid confounding results caused by drug activity against sporozoites in the blood. The second dose of P218 or placebo was administered 48 hours after the first dose (day 3) (Figure 1). Participants were confined to the unit until day 13, with an end of study outpatient visit at day 35 .

Blood samples for parasite assessments were taken immediately before PfSPZ inoculation and daily from day 7 to day 28. Plasmodium falciparum positivity was confirmed by Giemsa-stained thick blood smear when two unambiguous $P$. falciparum structures were seen in at least $0.5 \mu \mathrm{L}$ of blood, identified by two independent microscopists. However, positive microscopy was not required to trigger rescue therapy or used for outcomes assessment. Parasitemia was assessed at the Institute of Tropical Medicine, Antwerp, Belgium, using a quantitative PCR (qPCR) assay. DNA was extracted from $200 \mu \mathrm{L}$ of blood using the QIAamp 96 DNA Blood Kit (Qiagen, Hilden, Germany), and eluted in $200 \mu \mathrm{L}$ of water. Five microliters of DNA were used for qPCR analysis targeting the $P$. falciparum var gene acidic terminal sequence $(\approx 59$ copies per genome), as previously described. ${ }^{20}$ The limit of detection was 50 parasites $/ \mathrm{mL}$. Parasite densities were obtained by interpolating cycle thresholds from a standard curve prepared with titrated samples containing known numbers of infected erythrocytes diluted in whole blood $(10,000,000$ to 1 parasites/ $\mathrm{mL}$ ). Parasite positivity was defined as $\geq 250$ parasites $/ \mathrm{mL}$ blood. ${ }^{15,17}$ Immediately following detection of $\geq 250$ parasites/ $\mathrm{mL}$ blood or at day 28, artemether-lumefantrine (Riamet ${ }^{\circledR}$, Novartis Pharmaceuticals UK Ltd., London, UK) rescue therapy was administered plus single-dose primaquine (15 mg base) to ensure gametocyte clearance. In the case of contraindications or intolerance, atovaquone/proguanil (Malarone $^{\circledR}$, GlaxoSmithKline, Brentford, UK) could be given. Parasitemia by qPCR, thick blood smear, and malaria clinical score were evaluated before rescue treatment and at 24 hours and 72 hours following rescue treatment.

Malaria clinical score was assessed before PfSPZ inoculation, daily from day 7 until the day a participant was parasitemic, and at study end (day 35). Clinical adverse events consistent with malaria (myalgia, arthralgia, fatigue/lethargy, malaise, chills/shivering/rigors, sweating/hot spells, anorexia, nausea, vomiting, abdominal discomfort, fever, tachycardia, and hypotension) were scored as one (mild), two (moderate), or three (severe). These adverse events were classified as inoculum-related events and included in the malaria score only if the participant was concurrently parasitemic.

Frequent venous blood samples $(4 \mathrm{~mL})$ for PK assessments were taken in all cohorts. Bioanalysis was performed by Swiss BioQuant (Reinach, Switzerland), using a validated highperformance liquid chromatography tandem mass spectrometry method (analytical range $0.2 \mathrm{ng} / \mathrm{mL}$ to $200 \mathrm{ng} / \mathrm{mL}$ ). Pharmacokinetic parameters were derived from individual concentration-time data in plasma by SGS Life Sciences using Phoenix WinNonLin 8.0 (Pharsight Corporation, Palo Alto, CA). Drug exposure (area under the curve [AUC]) was calculated according to the linear up/log down trapezoidal method using actual sampling time points.

Adverse events were monitored continuously from informed consent until the last study-related activity and coded according to the Medical Dictionary for Regulatory Activities version 21.1. Vital signs were assessed and physical examinations performed throughout the study.

Blood samples were taken for biochemistry, hematology, coagulation testing, and serum folate measurement at 
screening, before study drug administration, on days 3 and 9 in cohort 1 and in cohorts 2 and 3 on days $3,8,10,14,21$, and 28 , and 1 day after parasitemia detection by qPCR. Troponin $T$ was evaluated at each parasitemia measurement to evaluate VIS model cardiac safety. ${ }^{21}$

Twelve-lead ECGs were performed at screening, before PfSPZ inoculation, before drug administration, at 2 hours and 12 hours after drug administration, on days 2 and 4 , then in cohort 1 on days 6/9, or in cohorts 2 and 3 in the event of parasitemia, on days 14-16, and on day 35. Recordings were performed once, except at screening, and before the first and second drug administration when they were performed in triplicate at 1-minute intervals.

Outcomes. The primary outcome for cohort 1 was the incidence, severity, and relationship of treatment-emergent adverse events during the 9-day observation period without PfSPZ VIS. The primary outcome for cohorts 2 and 3 was the cohort-specific, geometric mean time to parasitemia calculated as the time elapsed between PfSPZ inoculation and the first qPCR detected positive parasitemia until day 28 .

Secondary outcomes for cohort 1 were the estimation PK parameters for P218, P218 $\beta$-acyl glucuronide, $\mathrm{P} 218-\mathrm{OH}$, and $\mathrm{P} 218-\mathrm{OH} \beta$-acyl glucuronide. Pharmacokinetic parameters were estimated for parent P218 in cohorts 2 and 3 . In all three cohorts, secondary safety outcomes were changes from baseline in folate levels, hematology, clinical chemistry and urinalysis parameters, vital signs, and ECG parameters. Secondary safety outcomes for cohorts 2 and 3 were the incidence, severity, and relationship of treatment-emergent adverse events, the incidence and severity of malarial signs and symptoms, and the malaria clinical score at the time of rescue therapy administration.

Statistical methods. For this exploratory study, no formal sample size calculation was performed. However, if the nine P218-treated participants each in cohorts 2 and 3 did not develop positive parasitemia (qPCR $\geq 250$ parasites $/ \mathrm{mL}$ ) until day 28 , the protection rate for $\mathrm{P} 218$ would be $72 \%$ at $95 \%$ probability (lower limit of exact, one sided test, $95 \% \mathrm{Cl}: 0.72$ ). Statistical analyses were performed by SGS Life Sciences, using SAS ${ }^{\circledR}$ version 9.4 (SAS Institute Inc.). Safety was evaluated in all participants who received at least one dose of study drug. Pharmacokinetics were assessed in all randomized participants who received at least one dose of P218 and had at least one measurable concentration of parent and/or metabolite. Efficacy was examined for the intention-to-treat population, including all participants who received at least one dose of study drug and underwent PfSPZ VIS.

For the primary outcome in cohorts 2 and 3, time to positive parasitemia was analyzed using descriptive statistics, including the geometric mean and corresponding two-sided $90 \% \mathrm{Cls}$. For other outcomes, descriptive statistics were presented.

\section{RESULTS}

In vivo efficacy in a humanized mouse model and dose selection. In vivo efficacy in the $P$. falciparum "4-day" test is shown in Figure 2A. A nonlinear fitting to sigmoid doseresponse curve of $\log _{10}$ of $\%$ parasitemia at day 7 after infection versus the dose gave an estimated $\mathrm{ED}_{90}$ of $1.6 \mathrm{mg} / \mathrm{kg}$. A nonlinear fitting to sigmoid dose-response curve of $\log _{10}$ of $\%$ parasitemia at day 7 after infection versus the AUC of levels of P218 obtained during the first 23 hours after the first
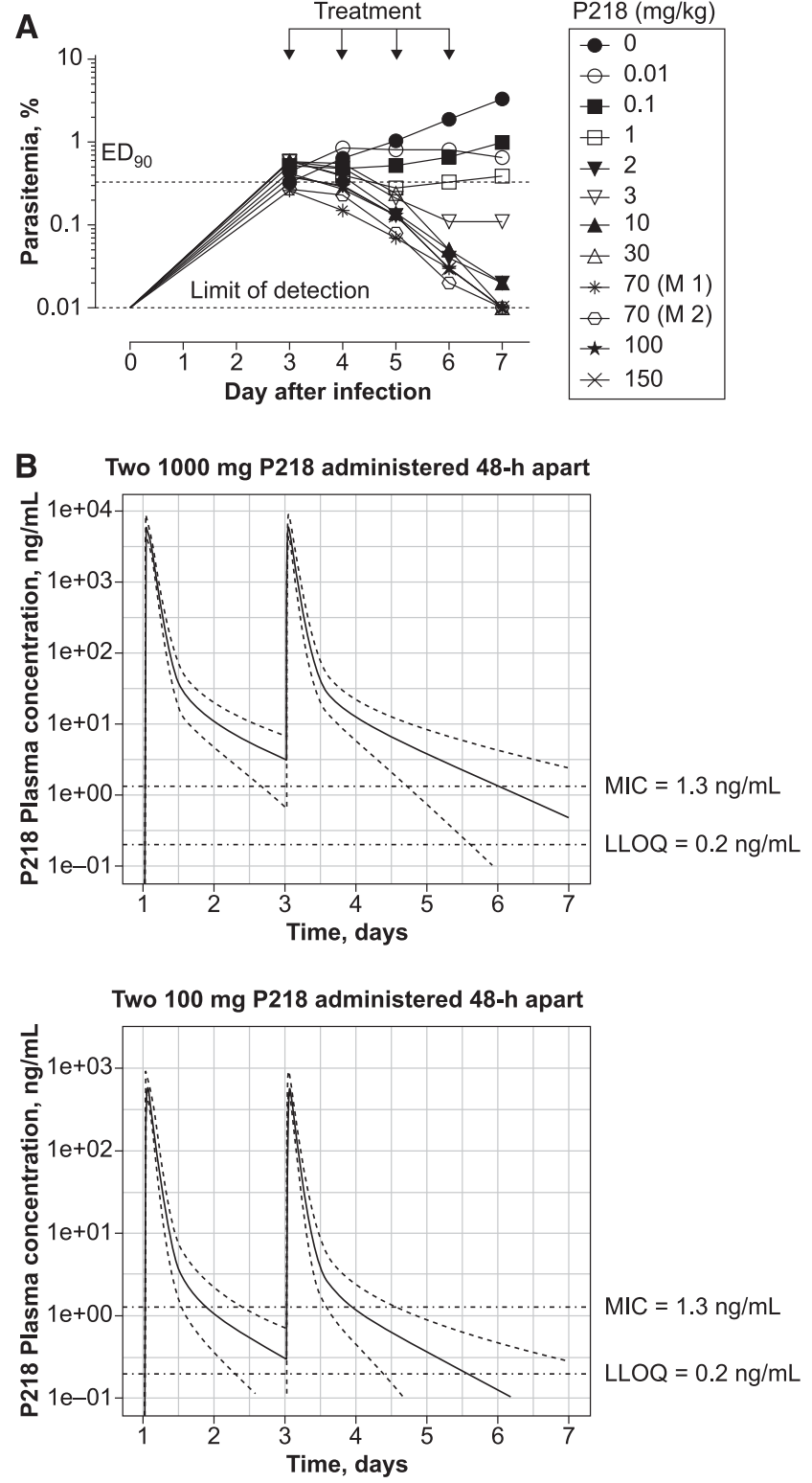

FIGURE 2. In vivo efficacy in a humanized mouse model and population pharmacokinetics for dose selection. (A) P218 oral efficacy in a standard 4-day test in female NOD-scid IL-2R ${ }^{\text {null }}$ mice engrafted with human erythrocytes and infected with $20 \times 10^{6}$ Plasmodium falciparum-infected erythrocytes ( $P$. falciparum Pf3D $7^{0087 / N 9}$ ). (B) Population PK simulations of P218 plasma concentration over time following oral administration of two $1,000 \mathrm{mg}$ doses administered 48 hours apart or two $100 \mathrm{mg}$ doses administered 48 hours apart (see also Supplemental Materials Methods S1). Data are median with fifth and 95th population percentiles generated from 1,000 trials of six participants each. MIC = minimum inhibitory concentration; LLOQ = lower limit of quantitation.

administration gave an estimated $A U C_{E D 90}$ of $0.1 \mu \mathrm{g} \cdot \mathrm{hour} / \mathrm{mL}$. The results were used to derive a target minimum parasiticidal concentration of $4.4 \mathrm{ng} / \mathrm{mL}$ and MIC of $1.3 \mathrm{ng} / \mathrm{mL}$.

Pharmacokinetic data from the completed first-in-human study with P218 were used to develop the popPK model. ${ }^{11}$ Using the mouse efficacy data, P218 popPK model simulations indicated that two single doses of $1,000 \mathrm{mg}$ of P218 administered 48 hours apart would maintain P218 plasma concentrations above the target mouse blood MIC for at least 

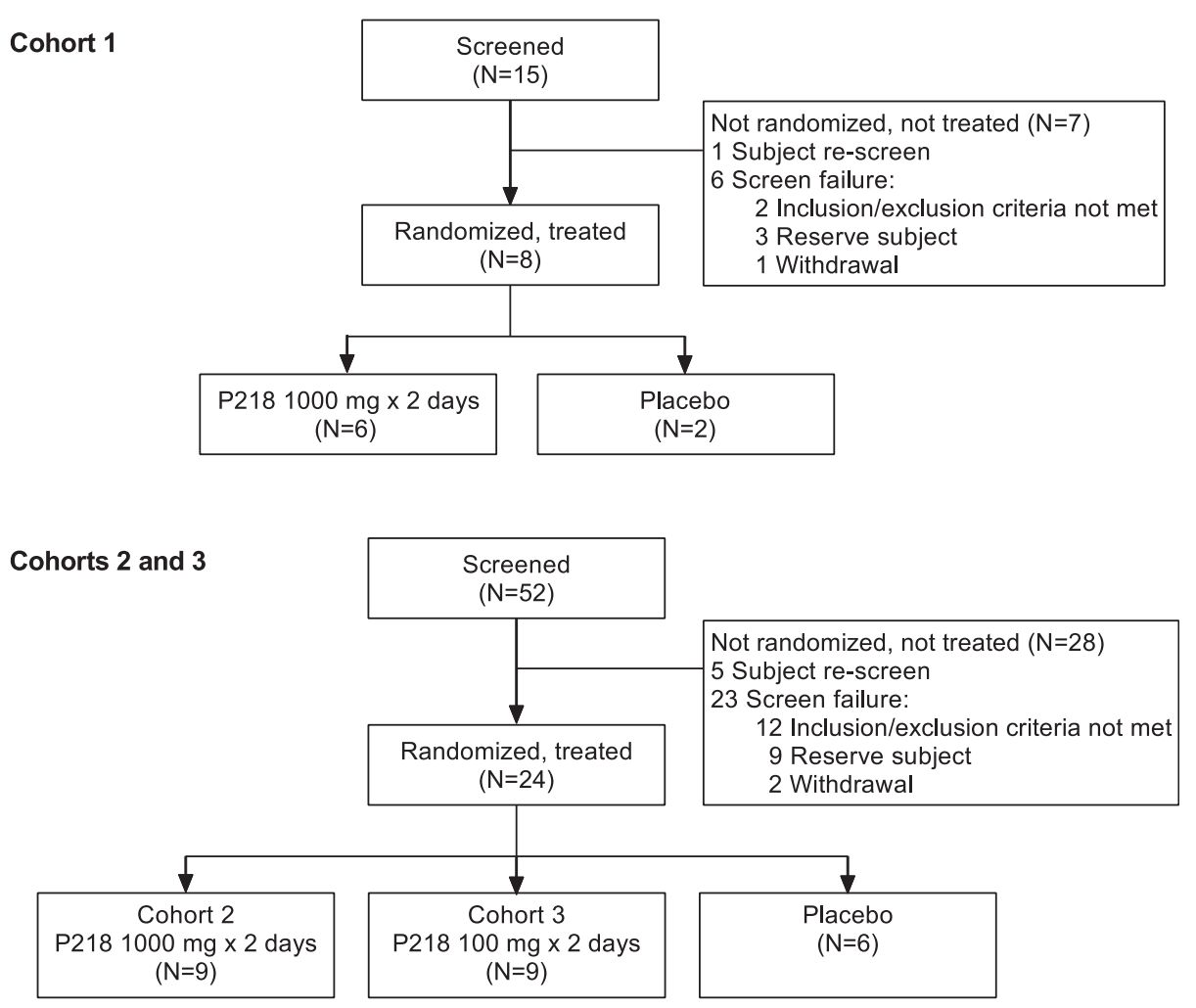

All randomized subjects in cohorts 1,2 and 3 completed the study

FIGURE 3. Participant disposition.

6 days (Figure 2B). The model predicted that there would be no accumulation of P218 or metabolites and similar exposure to that obtained with a single $1,000 \mathrm{mg}$ P218 dose. Two single doses of $100 \mathrm{mg}$ of P218 administered 48 hours apart were predicted to result in breakthrough parasitemia in some participants, allowing better estimation of the minimal inhibitory exposure of P218 required for chemoprotective activity (Figure 2B).

Participants. Of the 67 participants screened, 32 were enrolled, all of whom completed the study (Figure 3). Baseline demographic characteristics are shown in Table 1. All participants were included in the safety, PK, and efficacy analyses.

Efficacy. All participants receiving P218 remained parasite free in cohort $2(1,000 \mathrm{mg} \times 2$ days, $n=9)$, one became parasitemic in cohort 3 on day $15(100 \mathrm{mg} \times 2$ days, $n=9)$, and all participants in cohorts 2 and 3 who received placebo $(n=6)$ were parasitemic by day 12 (Figure 4). Although parasites were detected in one participant receiving $1,000 \mathrm{mg} \mathrm{P} 218$, this did not meet the protocol-defined threshold for parasitemia (Figure 4B). Geometric mean time to last assessment/positive parasitemia was 28.0 days with $\mathrm{P} 218$ in cohort 2 and 25.8 days (90\% Cl: 22.4-29.8) with P218 in cohort 3 compared with 10.6 days (90\% Cl: 9.9-11.4) with placebo (cohorts 2 and 3 ). The maximum recorded parasitemia level was 4,432 parasites/mL blood (Figure 4B).

Following the PfSPZ inoculation and intake of either P218 $100 \mathrm{mg}$ or $1,000 \mathrm{mg}$, no malaria signs and symptoms were reported. With placebo, malaria signs and symptoms were reported in 4/6 (66.7\%) participants (Supplemental Figure S1).

TABLE 1

Baseline demographic characteristics

\begin{tabular}{|c|c|c|c|c|c|}
\hline \multirow[b]{2}{*}{ Characteristic } & \multicolumn{2}{|c|}{ Cohort 1} & \multirow{2}{*}{$\begin{array}{l}\text { Cohorts } 2 \text { and } 3 \\
\text { Placebo }(n=6)\end{array}$} & \multirow{2}{*}{$\begin{array}{c}\text { Cohort } 2 \\
\begin{array}{c}\text { P218 1,000 } \mathrm{mg} / \text { day } \\
\times 2 \text { days }(n=9)\end{array}\end{array}$} & \multirow{2}{*}{$\begin{array}{c}\text { Cohort } 3 \\
\begin{array}{c}\text { P218 } 100 \mathrm{mg} / \text { day } \\
\times 2 \text { days }(n=9)\end{array}\end{array}$} \\
\hline & Placebo $(n=2)$ & $\begin{array}{l}\mathrm{P} 2181,000 \mathrm{mg} / \text { day } \\
\times 2 \text { days }(n=6)\end{array}$ & & & \\
\hline Median age (range) (years) & $24.5(24,25)$ & $33.5(24,45)$ & $34.5(33,42)$ & $36.0(22,40)$ & $35.0(23,42)$ \\
\hline Median height (range) $(\mathrm{cm})$ & $164.9(154.6,175.2)$ & $169.8(158.9,187.6)$ & $183.9(173.7,189.6)$ & $166.2(164.4,184.4)$ & $170.2(156.7,181.0)$ \\
\hline Median weight (range) $(\mathrm{kg})$ & $61.8(57.9,65.8)$ & $71.0(54.6,92.4)$ & $82.1(72.0,105.4)$ & $70.3(57.9,89.3)$ & $72.2(58.4,91.8)$ \\
\hline Median BMI (range) $\left(\mathrm{kg} / \mathrm{m}^{2}\right)$ & $22.8(21.4,24.2)$ & $24.6(21.6,26.3)$ & $25.5(21.0,29.3)$ & $24.9(21.1,29.6)$ & $23.5(20.8,28.3)$ \\
\hline \multicolumn{6}{|l|}{ Gender, $n(\%)$} \\
\hline Male & $1(50.0)$ & $3(50.0)$ & $6(100)$ & $5(55.6)$ & $5(55.6)$ \\
\hline Female & $1(50.0)$ & $3(50.3)$ & 0 & $4(44.4)$ & $4(44.4)$ \\
\hline \multicolumn{6}{|l|}{ Race, $n$ (\%) } \\
\hline White & $2(100)$ & $6(100)$ & $6(100)$ & $9(100)$ & $7(77.8)$ \\
\hline Other & 0 & 0 & 0 & 0 & $2(22.2)$ \\
\hline
\end{tabular}

* One Asian and one American Indian/Alaskan native. 

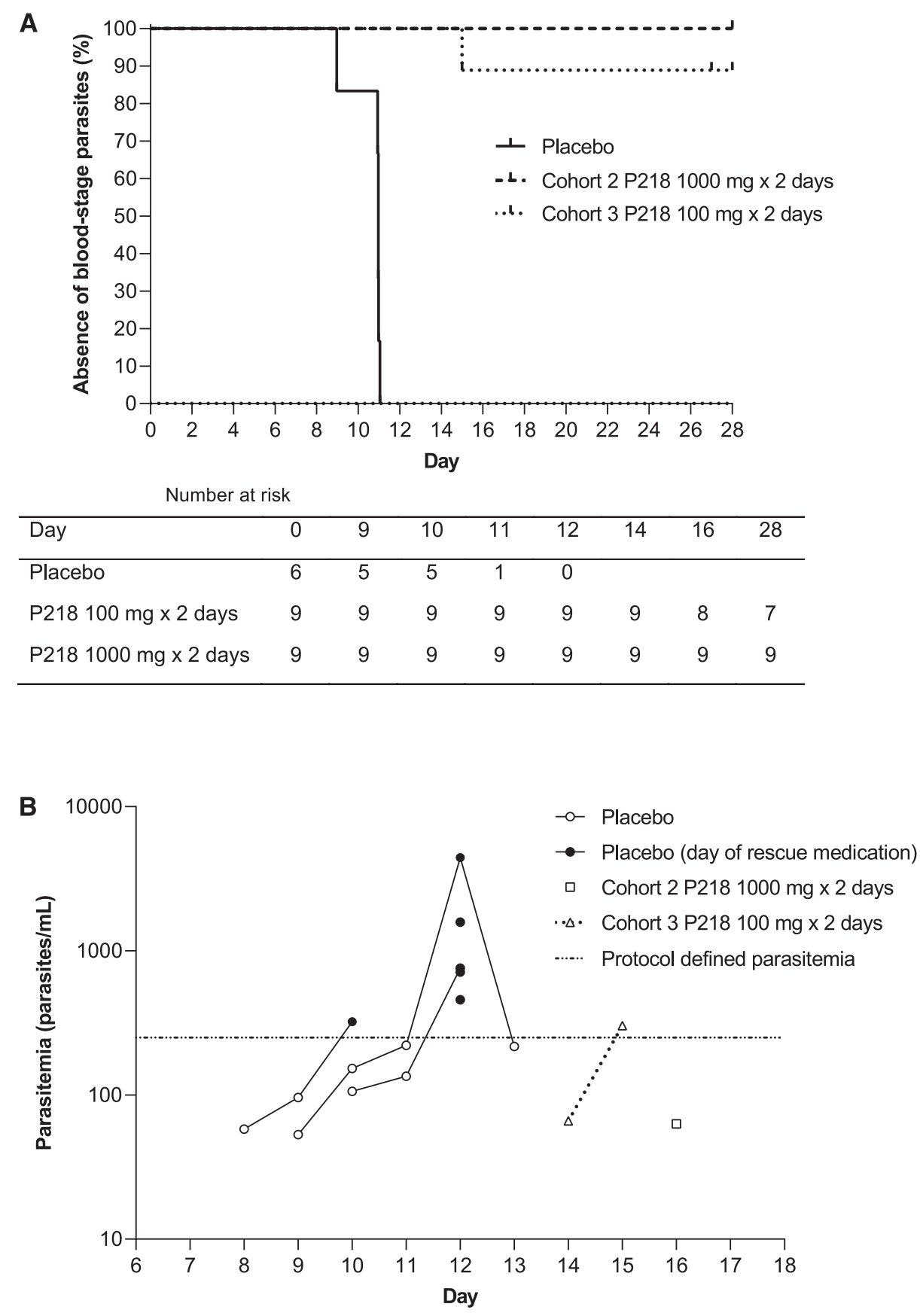

FIGURE 4. P218 chemoprotective efficacy. (A) Kaplan-Meier estimates of absence of detectable parasites with P218 chemoprevention following Plamodium falciparum sporozoite inoculation. One participant in cohort 3 had their final quantitative PCR (qPCR) assessment on day 27 and was censored at this point. (B) Parasitemia values from qPCR for individual participants following $P$. falciparum sporozoite inoculation following placebo, two doses of 1,000 mg P218 48 hours apart (cohort 2), or two doses of $100 \mathrm{mg}$ P218 48 hours apart (cohort 3). Protocol-defined positive parasitemia was $\geq 250$ parasites $/ \mathrm{mL}$ blood. Zero values are not shown. Closed symbols indicate the start of rescue therapy in the placebo group. Only one participant had a positive blood smear (placebo group day 9) with a corresponding qPCR parasite count of 257 parasites $/ \mathrm{mL}$ blood.

All reported malaria signs and symptoms were mild, with a maximum score of one per item and a maximum malaria clinical score of 5 .

Pharmacokinetics. P218 concentration-time profiles are shown in Figure 5A and PK parameters in Table 2. In healthy participants (cohort 1) and following PfSPZ inoculation (cohorts 2 and 3), P218 absorption was rapid; median $T_{\max }$ was 1 hour on day 1 and $1-1.75$ hours on day 3 . Following two P218 $1,000 \mathrm{mg}$ doses 48 hours apart, geometric mean $C_{\max }$ was similar on day 1 in cohort $1(6,640 \mathrm{ng} / \mathrm{mL})$ and cohort $2(6,769$ $\mathrm{ng} / \mathrm{mL})$ but on day 3 was slightly higher in cohort $1(6,860 \mathrm{ng} /$ $\mathrm{mL})$ versus cohort $2(6,090 \mathrm{ng} / \mathrm{mL})$. The $\mathrm{AUC}_{\mathrm{T}}$ on day 3 was also higher in cohort $1(16,426 \mathrm{ng} \cdot \mathrm{hour} / \mathrm{mL})$ versus cohort 2 (13,990 ng.hour/mL). For the participant with breakthrough parasitemia in cohort 3, P218 exposure was 1,365 ng.hour/mL on day 1 and $1,325 \mathrm{ng} \cdot \mathrm{hour} / \mathrm{mL}$ on day 3 , which was similar to the mean $\mathrm{AUC}_{\mathrm{T}}$ across cohort 3 on day $1(1,253 \mathrm{ng} \cdot \mathrm{hour} / \mathrm{mL})$ and day 3 (1,258 $\mathrm{ng} \cdot \mathrm{hour} / \mathrm{mL})$. 


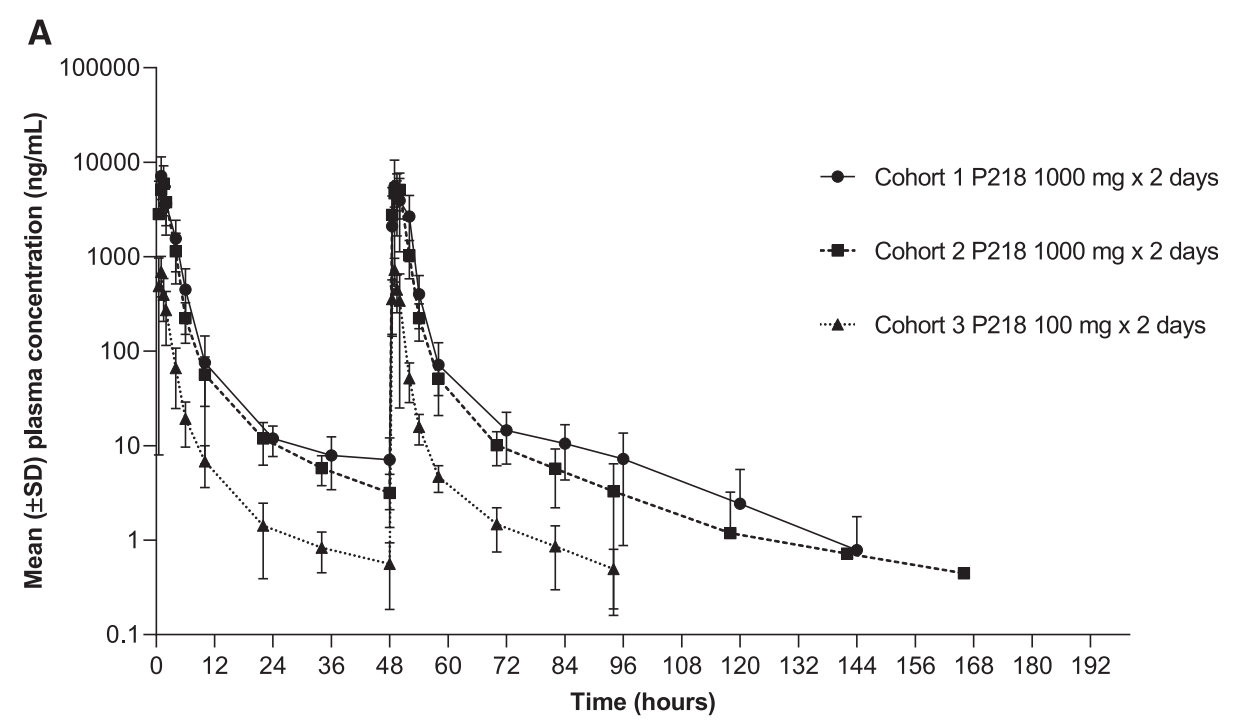

B

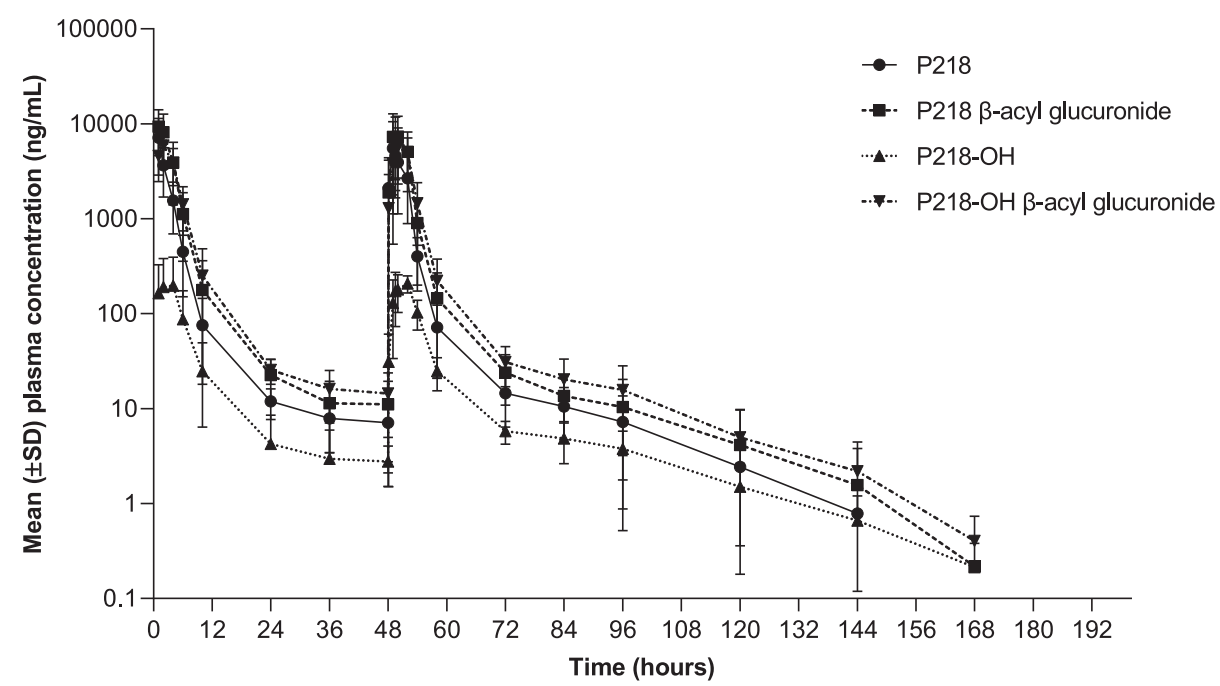

FIGURE 5. Mean plasma concentration-time profiles. (A) P218 after two doses of 1,000 mg P218 48 hours apart (cohort 1), or following Plasmodium falciparum sporozoite inoculation for two doses of 1,000 mg P218 48 hours apart (cohort 2), or two doses of $100 \mathrm{mg}$ P218 48 hours apart (cohort 3), and (B) P218 and metabolites following two doses of 1,000 mg P218 48 hours apart (cohort 1).

Across all cohorts, P218 accumulation between doses was negligible. P218 elimination was multiphasic, with a geometric mean $t_{1 / 2}$ following the second dose of 12.3 hours in cohort 1 , 11.9 hours in cohort 2 , and 11.2 hours in cohort 3 . Between the $100 \mathrm{mg}$ and 1,000 mg P218 doses, $C_{\max }$ increased by around 8-fold on days 1 and 3 , indicating a slightly less than doseproportional increase.

P218 metabolites formed rapidly after $1,000 \mathrm{mg}$ P218 administration on days 1 and 3 (Figure 5B, Table 3). P218 $\beta$-acyl glucuronide and $\mathrm{P} 218-\mathrm{OH} \beta$-acyl glucuronide were the most abundant metabolites. For all three metabolites, elimination was multiphasic, with $\mathrm{P} 218-\mathrm{OH}$ having the longest geometric mean $t_{1 / 2}$ at 21.1 hour versus 12.3 hours for $\mathrm{P} 218$.

Safety. Across all participants, there were no serious adverse events or deaths and no adverse events that resulted in study drug discontinuation. All adverse events were mild or moderate in severity. The most frequently reported adverse events occurring in participants who received P218 before rescue therapy administration were nasopharyngitis, headache, fatigue, and diarrhea (Table 4). Headache and diarrhea were the only adverse events to occur in all P218 treatment groups and not in the placebo group. Two adverse events were considered related to P218 treatment, one case of diarrhea (cohort 1) and one of headache (cohort 2). Following rescue therapy, $50.0 \%$ (3/6) of participants in the placebo group and $22.2 \%(2 / 9)$ in cohort 2 had adverse events (Supplemental Material Table S1).

In cohorts 2 and 3, adverse events related to PfSPZ inoculation in the placebo group were consistent with the symptoms of malaria. One participant in the placebo group had alanine aminotransferase $3.5 \times$ the upper limit of normal $(\mathrm{ULN}=49 \mathrm{U} / \mathrm{L}$; value $=170 \mathrm{U} / \mathrm{L})$ on day 21 , following 
TABLE 2

Plasma pharmacokinetic parameters for P218 after two doses of 1,000 mg P218 48 hours apart (cohort 1), or following Plasmodium falciparum sporozoite inoculation for two doses of 1,000 mg P218 48 hours apart (cohort 2), or two doses of $100 \mathrm{mg}$ P218 48 hours apart (cohort 3)

\begin{tabular}{|c|c|c|c|c|c|c|}
\hline \multirow[b]{3}{*}{ Pharmacokinetic parameter } & \multicolumn{2}{|c|}{ Cohort 1} & \multicolumn{2}{|c|}{ Cohort 2} & \multicolumn{2}{|c|}{ Cohort 3} \\
\hline & \multicolumn{2}{|c|}{$\mathrm{P} 2181,000 \mathrm{mg} /$ day $\times 2$ days $(n=6)$} & \multicolumn{2}{|c|}{$\mathrm{P} 2181,000 \mathrm{mg} /$ day $\times 2$ days $(n=9)$} & \multicolumn{2}{|c|}{ P218 $100 \mathrm{mg} /$ day $\times 2$ days $(n=9)$} \\
\hline & Day 1 & Day 3 & Day 1 & Day 3 & Day 1 & Day 3 \\
\hline $\mathrm{AUC}_{\mathrm{T}}$ (ng.hour/mL) & $15,871(34.4)$ & $16,426(32.4)$ & $13,662(17.9)$ & $13,990(24.1)$ & $1,253(28.7)$ & $1,258(28.5)$ \\
\hline $\mathrm{AUC}_{48 \text { hours-inf }}(\mathrm{ng} \cdot \mathrm{hour} / \mathrm{mL})$ & - & $16,590(31.9)$ & - & $13,445(20.6)^{\star}$ & - & 1,354 (28.8)† \\
\hline $\mathrm{AUC}_{48}$ hours-last (ng.hour/mL) & - & 16,579 (32.0) & - & 14,058 (24.3) & - & $1,258(28.6)$ \\
\hline$C_{\max }(\mathrm{ng} / \mathrm{mL})$ & $6,640(60.1)$ & 6,860 (73.9) & $6,769(39.5)$ & $6,090(31.9)$ & 793 (44.2) & 792 (28.8) \\
\hline$T_{\max }(\mathrm{h})$ & $1.0(1.0,4.0)$ & $1.75(1.0,4.0)$ & $1.0(0.6,1.5)$ & $1.5(1.0,2.0)$ & $1.0(0.5,1.9)$ & $1.0(0.5,2.0)$ \\
\hline$t_{1 / 2}(\mathrm{~h})$ & - & $12.3(61.4)$ & - & $11.9(106)^{\star}$ & - & $11.2(30.4) \dagger$ \\
\hline$R_{\mathrm{ac}}$ & - & $1.03(26.5)$ & - & $1.02(27.8)$ & - & 1.0 (16.6) \\
\hline $\mathrm{CL} / \mathrm{F}(\mathrm{L})$ & - & 60.3 (31.9) & - & $74.4(20.6)^{\star}$ & - & 73.8 (28.8)† \\
\hline$V_{z} / F(L)$ & - & 1,073 (65.4) & - & $1,282(97.4)^{\star}$ & - & 1,193 (20.1)† \\
\hline
\end{tabular}

administration of rescue medication on days 13 and 14 , which returned to normal by day 35 .

There were no clinically relevant differences in laboratory values with $\mathrm{P} 218$ versus placebo over time in any participant. There was no consistent pattern in the occurrence of abnormal laboratory values except that reticulocytes were more often high (54.2\% [13/24] versus $25.0 \%$ [2/8]) and folate levels were more often low $(41.7 \%$ [10/24] versus $12.5 \%$ [1/8]) in participants receiving P218 versus placebo (Supplemental Material Table S2). However, there was no clinically important decline in folate values in P218-treated participants and no consistent pattern of folate depletion (Supplemental Material Figure S2). There were no changes in troponin T or vital signs, and ECGs were clinically unremarkable for all participants (Supplemental Material Figure S3).

\section{DISCUSSION}

This study sought to characterize the safety and PK of a repeated $1,000 \mathrm{mg}$ or $100 \mathrm{mg} P 218$ dose in healthy volunteers and evaluate P218 chemoprotective potential following PfSPZ inoculation. P218 was administered 2 hours after sporozoite inoculation to ensure sufficient time for the sporozoites to reach the liver ( 45 minutes to 1 hour). Thus, any potential effect of P218 against sporozoites pre-hepatically en route to the liver was avoided. As P218 is also active against blood-stage parasites, ${ }^{9}$ our clinical study cannot distinguish between liverstage activity and activity against early schizonts emerging into the blood. P218 has a short half-life and is known to have greater activity against liver-stage versus blood-stage parasites. ${ }^{9,10}$ However, it is possible that the chemoprotective effect of P218 is conferred by a combined effect against liver-stage and blood-stage parasites. A VIS evaluating P218 against blood-stage parasites is being considered to further elucidate P218 stage-specific activity.

Emergence of parasitemia was suppressed in all participants in cohort 2 who received two 1,000 mg P218 doses 48 hours apart. One participant in cohort 2 had a parasite density of 63 parasites $/ \mathrm{mL}$ blood on day 16 , which did not meet the protocol-defined criteria for positive parasitemia. On repeated DNA extraction, no parasites could be found for this participant, and it is likely that the parasite density was too close to the limit of detection to be reliably determined. As P218 has in vivo activity against $P$. falciparum blood stages, it is possible that liver-stage efficacy in this participant was partial but that overt parasitemia was suppressed by residual drug. However, transient submicroscopic parasitemia has been observed in a previous VIS study evaluating chemoprotective efficacy. ${ }^{15}$

TABLE 3

Plasma pharmacokinetic parameters for P218 metabolites following two P218 doses of 1,000 mg 48 hours apart (cohort 1 [ $n=6$ ])

\begin{tabular}{|c|c|c|c|c|c|c|}
\hline \multirow[b]{2}{*}{ Pharmacokinetic parameter } & \multicolumn{2}{|c|}{ P218 $\beta$-acyl glucuronide } & \multicolumn{2}{|c|}{$\begin{array}{l}\mathrm{P} 218-\mathrm{OH} \\
-\end{array}$} & \multicolumn{2}{|c|}{ P218-OH $\beta$-acyl glucuronide } \\
\hline & Day 1 & Day 3 & Day 1 & Day 3 & Day 1 & Day 3 \\
\hline $\mathrm{AUC}_{\mathrm{T}}$ (ng $\cdot$ hour/mL) & $30,030(44.0)$ & $27,710(41.2)$ & 1,332 (15.6) & $1,404(10.4)$ & $25,464(29.1)$ & $26,076(29.9)$ \\
\hline $\mathrm{AUC}_{48 \text { hours-inf }}(\mathrm{ng} \cdot \mathrm{hour} / \mathrm{mL})$ & - & $27,958(41.1)$ & - & $1,508(9.3)$ & - & $26,403(29.9)$ \\
\hline $\mathrm{AUC}_{48 \text { hours-last }(\mathrm{ng} \cdot \text { hour } / \mathrm{mL})}$ & - & $27,945(41.1)$ & - & $1,498(9.2)$ & - & 26,395 (29.9) \\
\hline$C_{\max }(\mathrm{ng} / \mathrm{mL})$ & $10,330(38.1)$ & $10,208(53.4)$ & $253(28.1)$ & 248 (13.2) & $6,761(24.2)$ & 7,151 (31.4) \\
\hline$T_{\max }(\mathrm{h})$ & $1.5(1.0,4.0)$ & $1.75(1.0,4.0)$ & $3.0(1.0,4.0)$ & $4.0(1.5,4.0)$ & $2.0(1.0,4.0)$ & $3.0(1.5,4.0)$ \\
\hline$t_{1 / 2}(\mathrm{~h})$ & - & $12.1(57.7)$ & - & $21.1(50.2)$ & - & 14.9 (39.4) \\
\hline $\mathrm{R}_{\mathrm{ac}}$ & & $0.92(19.5)$ & - & $1.05(11.1)$ & - & $1.02(12.5)$ \\
\hline Metabolic ratio $\mathrm{AUC}_{\mathrm{T}}$ vs. $\mathrm{P} 218$ & $41.0(10.2)$ & $38.4(8.9)$ & $1.8(24.7)$ & $2.0(26.3)$ & $34.8(12.9)$ & $36.2(12.5)$ \\
\hline Metabolic ratio $\mathrm{AUC}_{48}$ hours-inf Vs. $\mathrm{P} 218$ & - & $38.3(9.0)$ & - & $2.1(25.3)$ & - & $36.2(12.5)$ \\
\hline
\end{tabular}


TABLE 4

Participants with adverse events of any cause following two doses of 1,000 mg P218 48 hours apart (cohort 1) or placebo without PfSPZ inoculation, and occurring after PfSPZ inoculation following two doses of 1,000 mg P218 48 hours apart (cohort 2), or two doses of $100 \mathrm{mg}$ P218 48 hours apart (cohort 3), or placebo (cohorts 2 and 3 ) before the initiation of rescue therapy

\begin{tabular}{|c|c|c|c|c|c|}
\hline \multirow[b]{2}{*}{ Adverse event, $n$ (\%) } & \multicolumn{2}{|c|}{ Cohort 1} & \multirow{2}{*}{ 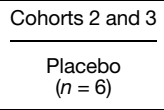 } & \multirow{2}{*}{$\begin{array}{c}\text { Cohort } 2 \\
\begin{array}{c}\text { P218 } 1,000 \mathrm{mg} / \text { day } \\
\times 2 \text { days }(n=9)\end{array}\end{array}$} & \multirow{2}{*}{$\begin{array}{c}\text { Cohort } 3 \\
\begin{array}{l}\text { P218 } 100 \mathrm{mg} / \text { day } \\
\times 2 \text { days }(n=9)\end{array}\end{array}$} \\
\hline & $\begin{array}{c}\text { Placebo } \\
(n=2)\end{array}$ & $\begin{array}{l}\mathrm{P} 2181,000 \mathrm{mg} / \text { day } \\
\times 2 \text { days }(n=6)\end{array}$ & & & \\
\hline Participants with any adverse event & $1(50.0)$ & $2(33.3)$ & $3(50.0)$ & $8(88.9)$ & $1(11.1)$ \\
\hline Headache & 0 & $1(16.7)$ & 0 & $2(22.2)$ & $1(11.1)$ \\
\hline Diarrhea & 0 & $1(16.7)$ & 0 & $1(11.1)$ & $1(11.1)$ \\
\hline Dizziness & 0 & $1(16.7)$ & $1(16.7)$ & 0 & 0 \\
\hline Lip dry & 0 & $1(16.7)$ & 0 & 0 & 0 \\
\hline Back pain & 0 & $1(16.7)$ & 0 & 0 & 0 \\
\hline Nasopharyngitis & $1(16.7)$ & 0 & 0 & $4(44.4)$ & 0 \\
\hline Fatigue & 0 & 0 & $1(16.7)$ & $3(33.3)$ & 0 \\
\hline Vulvovaginal candidiasis & 0 & 0 & 0 & $1(1.11)$ & 0 \\
\hline Chills & 0 & 0 & 0 & $1(11.1)$ & 0 \\
\hline Somnolence & 0 & 0 & 0 & $1(11.1)$ & 0 \\
\hline Joint stiffness & 0 & 0 & 0 & 1 (11.1) & 0 \\
\hline Musculoskeletal stiffness & 0 & 0 & 0 & $1(11.1)$ & 0 \\
\hline Skin wound & 0 & 0 & 0 & $1(11.1)$ & 0 \\
\hline Dysmenorrhea & 0 & 0 & 0 & $1(11.1)$ & 0 \\
\hline Hyperhidrosis & 0 & 0 & 0 & $1(11.1)$ & 0 \\
\hline Upper respiratory tract infection & 0 & 0 & 0 & 0 & $1(11.1)$ \\
\hline Pain in extremity & 0 & 0 & 0 & 0 & $1(11.1)$ \\
\hline Malaise & 0 & 0 & $1(16.7)$ & 0 & 0 \\
\hline Vessel puncture site hematoma & 0 & 0 & $1(16.7)$ & 0 & 0 \\
\hline
\end{tabular}

In cohort 3 ( $2 \times 100 \mathrm{mg}$ doses), one participant had breakthrough parasitemia with a maximum parasite density of 303 parasites/mL blood on day 15. However, no P218-treated participant had any signs or symptoms of malaria. These findings demonstrate the high chemoprotective potential of P218 against $P$. falciparum. By contrast, all participants who received placebo developed $\mathrm{PCR}$-defined parasitemia, and most had clinical manifestations of malaria. However, all malaria symptoms were mild, and PfSPZ-induced malaria was generally well tolerated, with no discontinuations for any reason. In all cases, rescue therapy rapidly cleared parasitemia without recrudescence.

The popPK model predicted that the short half-life of P218 relative to the $P$. falciparum liver cycle would lead to breakthrough parasitemia in all participants who received the $100 \mathrm{mg}$ P218 dose repeated after 48 hours. However, only one participant in this dosing group had breakthrough parasitemia. The striking chemoprotective activity of P218 limited the number of treatment failures and therefore precluded further PK/pharmacodynamic modeling. The popPK model predictions of the P218 dose needed for liver-stage chemoprotective efficacy were based on in vivo efficacy data against $P$. falciparum blood stages in a humanized mouse model. This was necessary as there is currently no validated in vivo model for determining candidate drug MICs against parasite liver stages. In vitro testing showed greater potency for P218 against liver stages versus blood stages. ${ }^{9,10}$ Hence, our popPK model was likely to overestimate the P218 dose required to successfully eliminate liver-stage parasites. This may have relevance to any study of potential chemoprotective agents where liver-stage activity exceeds blood-stage activity.

The finding that chemoprotective activity was high with P218 even at the $100 \mathrm{mg}$ dose suggests that the dose rationale based on the popPK model was in fact conservative. Recent additional preclinical evaluation indicates that P218 has potent activity against early $P$. falciparum liver stages (M. F.
Chughlay, personal communication). This might also explain why longer exposure to P218 was unnecessary to maintain efficacy in most of the participants in the low-dose group. Thus, the stage-specific activity against liver parasites may also affect the dosing regimen required for chemoprotective efficacy.

There were no safety concerns with P218, and all adverse events were mild or moderate in severity and self-limiting. P218 PK parameters and safety following P218 1,000 mg repeat dose were similar to those formerly noted for single-dose administration, ${ }^{11}$ with minimal accumulation between doses. Also, PK parameters for P218 metabolites were similar following two $1,000 \mathrm{mg}$ doses versus those previously reported for a single $1,000 \mathrm{mg}$ dose. ${ }^{11}$ There was good correspondence between the PK parameters for P218 and metabolites following two doses and the popPK prediction. Overall, there were no clinically relevant differences between P218 PK in cohort 1 versus cohort 2 following PfSPZ inoculation. For the one participant with a reported positive parasitemia in cohort 3, P218 exposure was not markedly different compared with exposure in the rest of the cohort. Thus, it is likely that the $100 \mathrm{mg}$ repeat dose was approaching the limit of efficacy, although it was still able to clear parasites in most of the participants.

Antimalarial drugs targeting PfDHFR are known to deplete folate levels and cause gastrointestinal symptoms. ${ }^{22-24}$ Plasma and serum folate levels are susceptible to changes in dietary intake of folate, and a single measurement cannot distinguish between a transient drop in folate intake and folate depletion. ${ }^{25,26}$ In this study, repeated serum folate measurements were taken over the study duration to identify any concerning trends in folate depletion. Although folate levels were more likely to be low in the P218-treated participants versus those receiving placebo, there was no trend over time for folate depletion, or evidence of a dose-response between the $100 \mathrm{mg}$ and 1,000 mg P218 doses. Diarrhea was reported for $12.5 \%$ (3/24) of participants receiving P218 versus none for placebo, and all three cases occurred in female participants 
with low folate levels (5.4-8.4 nmol/L; lower limit of normal 8.8 $\mathrm{nmol} / \mathrm{L})$. The first-in-human study also found no trend for serum folate reductions with $\mathrm{P} 218$ and no relationship to dose over the range $10-1,000 \mathrm{mg}$, but there were no cases of diarrhea. ${ }^{11}$ As doses of up to $1,000 \mathrm{mg} \mathrm{P} 218$ have been tested, the lack of a definitive effect on folate is encouraging. However, the data obtained so far do not exclude the possibility of an effect of P218 on folate, and more detailed evaluations are being considered for subsequent clinical studies. Also, the findings so far include a limited number of doses in healthy participants undergoing VIS and cannot be extrapolated to the situation in African children and pregnant women exposed to $P$. falciparum, who may also be malnourished or suffering from anemia while being exposed to steady-state drug concentrations over a malaria season. Thus, the effect of P218 clinical formulations on folate levels in the relevant populations for chemoprevention requires careful assessment.

The short half-life of P218 represents a barrier to effective chemoprotection in malaria-endemic areas where long halflife drugs are targeted to allow full coverage during the malaria season. However, the possibility of developing a long-acting injectable depot formulation is being investigated. This would allow P218 extended release, with the aim of maintaining chemoprotective drug levels over at least 1 week, and preferably over 1 month, following a single injection. The current study provides proof of antimalarial pharmacodynamics in humans for P218 and information on the target plasma blood levels needed for human chemoprotective efficacy. However, chemoprotective efficacy of the eventual clinical formulation will require confirmation.

P218 showed excellent chemoprotective efficacy in this study, and two $1,000 \mathrm{mg}$ doses 48 hours apart had good safety/tolerability, with no concerning findings. The current data clinically confirm P218 chemoprotective activity against $P$. falciparum and support the further development of P218 for malaria chemoprotection.

Received September 9, 2020. Accepted for publication December 2, 2020.

Published online February 8, 2021.

Note: Supplemental information, table, and figures appear at www.ajtmh.org.

Acknowledgments: We gratefully acknowledge the contribution of the volunteers who participated in this study and those who participated in the first malaria VIS in Belgium at the SGS Life Sciences Clinical Pharmacology Unit in Antwerp, Belgium. Researchers at GlaxoSmithKline (Tres Cantos) conducted the in vivo efficacy experiments informing dose selection, and we acknowledge the contributions of Belén Jiménez-Díaz, Helena Garuti, Lorena Cortés, Vanessa Gómez, Maria Santos Martínez, Santiago Ferrer, and Leticia Huertas. We acknowledge Janssen Pharmaceuticals, Belgium, and BIOTEC, Thailand, for their ongoing collaboration with Medicines for Malaria Venture (MMV) in the development of P218. Also, we thank Yongyuth Yuthavong (BIOTEC), Tim Wells, Stephan Duparc, and Jörg Möhrle (MMV).

Financial support: This study was sponsored by Medicines for Malaria Venture, Geneva, Switzerland. Funding for this study was provided by MMV and Janssen Pharmaceuticals. Naomi Richardson of Magenta Communications Ltd was funded by Medicines for Malaria Venture.

Disclosures: M. F. C., M. E. G., C. D., B. C., M. H. C.-R., and S. C. are employees of Medicines for Malaria Venture. P.-J. B. and A. L. are employees of SGS Life Sciences. M. W. M. is an employee of ICON Clinical Research GmbH. I. A.-B. was an employee of GlaxoSmithKline at the time of the study and is currently employed by the Art of
Discovery. S. V. is an employee of GlaxoSmithKline. G. L. is an employee of PTx Solutions. A. R.-U. is an employee of the Institute of Tropical Medicine, Antwerp. J.-P. V. G. is an employee of the University of Antwerp. SGS Life Sciences, ICON plc, PTx Solutions, the Institute of Tropical Medicine, and the University of Antwerp, all received support from Medicines for Malaria Venture related to the conduct of this study. Naomi Richardson of Magenta Communications Ltd wrote the first draft of this article from the statistical output, provided editorial support, collated author contributions, and provided graphic services. Named authors employed by Medicines for Malaria Venture were involved in the design, execution, analysis, interpretation, and reporting of the study as detailed in the author contributions.

Authors' addresses: Mohamed Farouk Chughlay, Myriam El Gaaloul, Cristina Donini, Brice Campo, Mohammed H. Cherkaoui-Rbati, and Stephan Chalon, Medicines for Malaria Venture (MMV), Geneva, Switzerland, E-mails: chughlayf@mmv.org, elgaaloulm@mmv.org, doninic@mmv.org, campob@mmv.org, cherkaouim@mmv.org, and chalons@mmv.org. Pieter-Jan Berghmans and Alexander Lucardie, SGS Life Sciences, Clinical Pharmacology Unit, Antwerpen, Belgium, E-mails: pieterjan.berghmans@sgs.com and alexander.lucardie@ sgs.com. Michael W. Marx, ICON Clinical Research GmbH, Langen, Germany, E-mail: michael.marx@iconplc.com. Grant Langdon, PTx Solutions, London, United Kingdom, E-mail: grant@ptxsolutions.co.uk. Iñigo Angulo-Barturen, The Art of Discovery, Biscay Science and Technology Park, Basque Country, Spain, E-mail: inigo.ab@tadmed.com. Sara Viera, GlaxoSmithKline R\&D (GSK), Madrid, Spain, E-mail: sara.m.viera@gsk.com. Anna Rosanas-Urgell, Institute of Tropical Medicine, Antwerp, Belgium, E-mail: arosanas@itg.be. Jean-Pierre Van Geertruyden, Global Health institute, University of Antwerp, Antwerpen, Belgium, E-mail: jean-pierre.vangeertruyden@ uantwerpen.be.

This is an open-access article distributed under the terms of the Creative Commons Attribution (CC-BY) License, which permits unrestricted use, distribution, and reproduction in any medium, provided the original author and source are credited.

\section{REFERENCES}

1. WHO, 2019. World Malaria Report. Geneva, Switzerland: World Health Organization. Available at: https://www.who.int/ publications-detail/world-malaria-report-2019. Accessed 31 July, 2020.

2. Stevens GA, Finucane MM, De-Regil LM, Paciorek CJ, Flaxman SR, Branca F, Pena-Rosas JP, Bhutta ZA, Ezzati M; Nutrition Impact Model Study Group, 2013. Global, regional, and national trends in haemoglobin concentration and prevalence of total and severe anaemia in children and pregnant and non-pregnant women for 1995-2011: a systematic analysis of populationrepresentative data. Lancet Glob Health 1: e16-e25.

3. Stanisic DI et al., 2015. Risk factors for malaria and adverse birth outcomes in a prospective cohort of pregnant women resident in a high malaria transmission area of Papua New Guinea. Trans $R$ Soc Trop Med Hyg 109: 313-324.

4. Matangila JR, Mitashi P, Inocencio da Luz RA, Lutumba PT, Van Geertruyden JP, 2015. Efficacy and safety of intermittent preventive treatment for malaria in schoolchildren: a systematic review. Malar J 14: 450.

5. WHO Global Malaria Programme, WHO Department of Reproductive Health and Research, WHO Department of Maternal N, Child and Adolescent Health, 2013. WHO Policy Brief for the Implementation of Intermittent Preventive Treatment of Malaria in Pregnancy Using Sulfadoxine-Pyrimethamine (IPTp-SP). Geneva, Switzerland: World Health Organization. Available at: https://www.who.int/malaria/publications/atoz/iptp-spupdated-policy-brief-24jan2014.pdf?ua=1. Accessed July 31, 2020.

6. WHO, 2010. WHO Policy Recommendation on Intermittent Preventive Treatment during Infancy with SulphadoxinePyrimethamine (IPTi-SP) for Plasmodium falciparum Malaria Control in Africa. Geneva, Switzerland: World Health Organization. Available at: https://www.who.int/malaria/news/WHO_ policy_recommendation_IPTi_032010.pdf?ua $=1$. Accessed July 31, 2020. 
7. WHO Global Malaria Programme, 2012. WHO Policy Recommendation: Seasonal Malaria Chemoprevention (SMC) for Plasmodium falciparum Malaria Control in Highly Seasonal Transmission Areas of the Sahel Sub-region in Africa. Geneva, Switzerland: World Health Organization. Available at: https://www. who.int/malaria/publications/atoz/smc_policy_recommendation_ en_032012.pdf?ua=1. Accessed July 31, 2020.

8. Nkoli Mandoko P et al., 2018. Prevalence of Plasmodium falciparum parasites resistant to sulfadoxine/pyrimethamine in the Democratic Republic of the Congo: emergence of highly resistant pfdhfr/pfdhps alleles. J Antimicrob Chemother 73: 2704-2715.

9. Yuthavong $Y$ et al., 2012. Malarial dihydrofolate reductase as a paradigm for drug development against a resistancecompromised target. Proc Natl Acad Sci U S A 109: $16823-16828$.

10. Roth A et al., 2018. A comprehensive model for assessment of liver stage therapies targeting Plasmodium vivax and Plasmodium falciparum. Nat Commun 9: 1837.

11. Chughlay MF, Rossignol E, Donini C, El Gaaloul M, Lorch U, Coates S, Langdon G, Hammond T, Möhrle J, Chalon S, 2020. First-in-human clinical trial to assess safety, tolerability and pharmacokinetics of P218, a novel candidate for malaria chemoprotection. Br J Clin Pharmacol 86: 1113-1124.

12. Nzila A, 2006. The past, present and future of antifolates in the treatment of Plasmodium falciparum infection. J Antimicrob Chemother 57: 1043-1054.

13. Ockenhouse CF et al., 2015. Ad35.CS.01-RTS,S/AS01 heterologous prime boost vaccine efficacy against sporozoite challenge in healthy malaria-naive adults. PLoS One 10: e0131571.

14. Gomez-Perez GP et al., 2015. Controlled human malaria infection by intramuscular and direct venous inoculation of cryopreserved Plasmodium falciparum sporozoites in malaria-naive volunteers: effect of injection volume and dose on infectivity rates. Malar J 14: 306

15. Sulyok $M$ et al., 2017. DSM265 for Plasmodium falciparum chemoprophylaxis: a randomised, double blinded, phase 1 trial with controlled human malaria infection. Lancet Infect Dis 17: 636-644.

16. Jongo SA et al., 2018. Safety, immunogenicity, and protective efficacy against controlled human malaria infection of
Plasmodium falciparum sporozoite vaccine in Tanzanian adults. Am J Trop Med Hyg 99: 338-349.

17. Murphy SC et al., 2018. A randomized trial evaluating the prophylactic activity of DSM265 against preerythrocytic Plasmodium falciparum infection during controlled human malarial infection by mosquito bites and direct venous inoculation. $J$ Infect Dis 217: 693-702.

18. Angulo-Barturen I et al., 2008. A murine model of falciparummalaria by in vivo selection of competent strains in nonmyelodepleted mice engrafted with human erythrocytes. PLoS One 3: e2252.

19. Jimenez-Diaz MB et al., 2009. Improved murine model of malaria using Plasmodium falciparum competent strains and nonmyelodepleted NOD-scid IL2Rgammanull mice engrafted with human erythrocytes. Antimicrob Agents Chemother 53: 4533-4536.

20. Hofmann N, Mwingira F, Shekalaghe S, Robinson LJ, Mueller I, Felger I, 2015. Ultra-sensitive detection of Plasmodium falciparum by amplification of multi-copy subtelomeric targets. PLoS Med 12: e1001788.

21. Marrelli MT, Brotto $M, 2016$. The effect of malaria and anti-malarial drugs on skeletal and cardiac muscles. Malar J 15: 524.

22. Shiroky JB et al., 1993. Low-dose methotrexate with leucovorin (folinic acid) in the management of rheumatoid arthritis. Results of a multicenter randomized, double-blind, placebo-controlled trial. Arthritis Rheum 36: 795-803.

23. Shea B, Swinden MV, Ghogomu ET, Ortiz Z, Katchamart W, Rader T, Bombardier C, Wells GA, Tugwell P, 2014. Folic acid and folinic acid for reducing side effects in patients receiving methotrexate for rheumatoid arthritis. J Rheumatol 41: 1049-1060.

24. Nzila A, Okombo J, Molloy AM, 2014. Impact of folate supplementation on the efficacy of sulfadoxine/pyrimethamine in preventing malaria in pregnancy: the potential of 5-methyltetrahydrofolate. J Antimicrob Chemother 69: 323-330.

25. Snow CF, 1999. Laboratory diagnosis of vitamin B12 and folate deficiency: a guide for the primary care physician. Arch Intern Med 159: 1289-1298.

26. Green R, 2011. Indicators for assessing folate and vitamin B-12 status and for monitoring the efficacy of intervention strategies. Am J Clin Nutr 94: 666S-72S. 